\title{
Structural Diversity of Aspergillus (Section Nigri) Spores
}

\author{
Marta Filipa Simões, Cledir Santos, and Nelson Lima* \\ IBB-Institute for Biotechnology and Bioengineering, Centre of Biological Engineering, Universidade do Minho, \\ Campus de Gualtar, 4710-057 Braga, Portugal
}

\begin{abstract}
The taxonomy of fungal species, similar to that of many other microorganisms, suffers frequent revisions due to the discovery of new species and to the development and gathering of characterization data and morphological information. Morpho-taxonomy helps in the identification of many species. This work presents the macro, micro-morphological, and spectral mass analyses for phenotypic characterization of 13 species of Aspergillus section Nigri, showing that the characterization of spores (conidia) by scanning electron microscopy can be used as a tool to discriminate key morphological characteristics and separate closely related fungi. These results were corroborated by colony plates, stereomicroscopy, light microscopy, and spectral mass data.
\end{abstract}

Key words: Aspergillus section Nigri, fungal taxonomy, morpho-taxonomy, spore characterization

\section{INTRODUCTION}

Species of Aspergillus section Nigri (known as black aspergilli) have been extensively used for various biotechnological purposes and are among the best-studied fungi. Many are responsible for biodeterioration of diverse commodities, including food (Abarca et al., 2004). The Nigri section has evolved continuously, and the last taxonomy revision includes 26 different taxa (Varga et al., 2011). However, because of molecular problems detected on $\beta$-tubulin DNA primers by Hubka and Loralik (2012), Aspergillus japonicus should be treated as a synonym of Aspergillus violaceofuscus, reducing the number to 25 different taxa. Similarly, Aspergillus fijiensis, described by Varga et al. (2011) as a new species, was found to be the already known Aspergillus brunneoviolaceus. Meanwhile, two more species were described by Jurjević et al. (2012), which means that we currently accept 27 different taxa.

The identification of species is a fundamental goal in microbiology. Information about each microorganism, such as morphological description, physiological and biochemical properties, ecological roles, and societal risks or benefits, is a key element in this process.

The concept of a distinct species is clearly abstract; delimitations are very difficult and often not consensual. Identification of fungi, even for experts, can be timeconsuming and is hampered by frequent revisions of the taxonomic schemes. In addition, each taxonomic group has specialized literature, terminology, and characters. Some practical solutions have been designed to overcome these limitations with very limited success (Paterson et al., 2004, 2006). This occurs to the extent that identifications can only be undertaken with a degree of reliability, by a small number of scientists skilled in the "art."

The black aspergilli are considered a taxonomically challenging group of fungi. It is difficult to recognize some of these species. Several approaches have been developed to

Received December 26, 2012; accepted April 29, 2013

*Corresponding author. E-mail: nelson@ie.uminho.pt this end, such as new taxonomic schemes using extensive molecular analysis (de Vries et al., 2005; Varga et al., 2011), and all available information is valuable in differentiating each strain. Therefore, a more complete and polyphasic approach allows better results in fungal taxonomy studies (Varga et al., 2011; Simões et al., 2013). The aim of this study was to contribute additional information on the existing strains of the section Nigri from the genus Aspergillus.

\section{Materials and Methods}

Thirteen strains belonging to Aspergillus section Nigri were selected and supplied by the Micoteca da Universidade do Minho (MUM) fungal culture collection (Table 1). For the macro-morphological analysis, the selected strains were grown on potato dextrose agar (PDA, OXOID CM0139, Basingstoke Hampshire, UK), malt extract agar (MEA, malt extract $2 \%$, mycological peptone $0.1 \%$, glucose $2 \%$, and agar 2\%), and Czapek yeast extract agar (CYA, sucrose 2\%, yeast extract $0.5 \%, \mathrm{NaNO}_{3} 0.3 \%, \mathrm{KCl} 0.05 \%, \mathrm{MgSO}_{4} \cdot 7 \mathrm{H}_{2} \mathrm{O}$ $0.05 \%, \mathrm{FeSO}_{4} \cdot 7 \mathrm{H}_{2} \mathrm{O} 0.001 \%, \mathrm{~K}_{2} \mathrm{HPO}_{4} 0.1 \%$, and agar $2 \%$ ). To guarantee the same medium depth in all of the plates and a standardized growth, $20 \mathrm{~mL}$ of medium was poured into standard $(90 \mathrm{~mm})$ sterile and disposable Petri dishes. Each plate was inoculated at three points, equidistant from the center, and incubated in the dark at $25^{\circ} \mathrm{C}$ for 7 days. To prevent stray colonies on the plates, inoculation was made from a spore suspension on a solution of $0.2 \%$ agar with $0.05 \%$ tween 80 . For microscopy analysis the strains were grown for 3 or 4 days on MEA.

Digital images of colonies were obtained on a register recording system for fungal plates that consists of a dedicated macro-photo stand with a Canon EOS 5D Mark II camera. The camera was operated with a remote shutter release device equipped with a $150-\mathrm{mm}$ interchangeable Sigma (St. Louis, MO, USA) lens APO MACRO F2.8EX DG HSM. The equipment consisted of a Polaroid $\mathrm{MP}^{+}$land camera stand where the camera was held horizontally at bull's eye level and pointing down toward the culture plates 
Table 1. List of Strains Selected for This Study.

\begin{tabular}{|c|c|c|c|}
\hline Species & Isolate Number & Geographical Origin & Substrate \\
\hline A. aculeatus & $\begin{array}{l}\text { MUM 03.11 } \\
\left(=\text { NRRL } 5094^{\mathrm{T}}\right)^{\mathrm{a}}\end{array}$ & Unknown & Tropical soil \\
\hline A. brasiliensis & MUM 06.179 & Portugal & Grapes Cabernet Sauvignon \\
\hline A. carbonarius & MUM 01.08 & Unknown & Unknown \\
\hline A. ellipticus & $\begin{array}{l}\text { MUM } 03.12^{\mathrm{T}} \\
\left(=\text { NRRL } 5120^{\mathrm{T}}\right)\end{array}$ & Costa Rica & Soil \\
\hline A. ibericus & MUM 04.68 & Portugal & Grapes Aragonês \\
\hline A. japonicus & $\begin{array}{l}\text { MUM } 98.03 \\
(=\text { DSM 2345) }\end{array}$ & Unknown & Unknown \\
\hline A. lacticoffeatus & $\begin{array}{l}\text { MUM } 06.150^{\mathrm{T}} \\
\left(=\mathrm{CBS} 101883^{\mathrm{T}}\right)^{\mathrm{c}}\end{array}$ & Indonesia & Coffee robusta (Rubiaceae) \\
\hline A. niger & MUM 05.13 & Portugal & Grapes Aragonês \\
\hline A. phoenicis & $\begin{array}{l}\text { MUM } 03.05 \\
(=\text { NRRL 365) }\end{array}$ & Unknown & Unknown \\
\hline A. sclerotioniger & $\begin{array}{l}\text { MUM 06.151 } \\
\left(=\text { CBS } 115572^{\mathrm{T}}\right)\end{array}$ & India & Arabic coffee \\
\hline A. tubingensis & $\begin{array}{l}\text { MUM 06.152 } \\
\left(=\text { CBS } 134.48^{\mathrm{T}}\right)\end{array}$ & Unknown & Unknown \\
\hline A. uvarum & MUM 08.01 & Portugal & Grapes Tinta Barroca \\
\hline A. vadensis & $\begin{array}{l}\text { MUM } 06.153^{\mathrm{T}} \\
\left(=\text { CBS } 113365^{\mathrm{T}}\right)\end{array}$ & Unknown & Dead plant tissue \\
\hline
\end{tabular}

${ }^{a}$ Northern Regional Research Laboratory

${ }^{\mathrm{b}}$ Deutsche Sammlung von Mikroorganismen

${ }^{\mathrm{c} C e n t r a a l b u r e a u}$ voor Schimmelcultures

using a constant distance of $89 \mathrm{~cm}$. Two $45^{\circ}$ oblique lighting arms, each holding a cool fluorescent LED-500 (WalimexPro, Burgheim, Germany) with $630 \mathrm{~lx}$ and $6200 \mathrm{~K}$ color temperature for reflected light and a white light panel (LP-400), were used as a baseboard for transmitted light. The aperture and depth of field were constants and the optimal focus was reached using an ad-hoc optimization. All photographs were acquired using EOS utility software (Canon, Reigate, Surrey, UK) for acquisition of images and were saved in an uncompressed file.

To have a clear image comparison of the size of the conidial head, a stereomicroscope (Leica MZ12.5, Heerburgg, Switzerland) equipped with a digital camera (Leica DC 150, PAL) was used. Fungal characterization was continued on a light microscope [Leica DM R, equipped with a digital camera (Leica DC 150, PAL)] and a scanning electron microscope [NanoSEM-FEI Nova ${ }^{\mathrm{TM}} 200$ (FEG/SEM); EDAXPegasus X4M (EDS/EBSD)]. For both the stereomicroscope and light microscope, Leica LAS EZ V1.7.0 software was used for acquisition and analysis of images.

Samples for light microscopy were mounted on a slide, abundantly washed with alcohol $(96 \%)$ to remove excessive spores, and stained with a lactophenol blue solution.

For scanning electron microscopy (SEM), a drop of medium (MEA) was mounted on a SEM stub under aseptic conditions. The stub was inoculated and incubated in the dark at $25^{\circ} \mathrm{C}$. After visible fungal growth, the sample was directly covered with a mixture of gold and platinum (80/ $20 \%$ ) for posterior analysis. Each single image was digitally produced and registered at variable magnifications.
Samples for spectral analysis by matrix-assisted laser desorption/ionization time-of-flight mass spectrometry (MALDI-TOF MS, AXIMA LNR Shimadzu, Kratos Analytical, Manchester, UK) were grown in MEA and incubated in the dark at $25^{\circ} \mathrm{C}$ for 4 days. The procedures were followed as described by Rodrigues et al. (2011). Briefly, for the flex target plate preparation, $\sim 1 \mu \mathrm{g}$ of spores and young mycelium of each species was transferred directly from the culture plate to the 48-well MALDI-TOF plate. Immediately thereafter, $0.5 \mu \mathrm{L}$ of matrix solution $[7.5 \% 2,5$ dihydroxybenzoic acid in ethanol/water/acetonitrile (1:1:1) with $0.03 \%$ trifluoroacetic acid] was added to the samples and mixed gently. The sample mixtures were air-dried at room temperature. Each sample was spotted in duplicate to test reproducibility. During the analyses, all solutions were prepared and stored at $4^{\circ} \mathrm{C}$. Data were analyzed on the basis of a matrix of pairwise correlation values for spectra after smoothing baseline corrections and peak detections. The peak lists of the selected strains were directly transferred into SARAMIS ${ }^{\circledR}$ (AnagnosTec, Potsdam, Germany) software.

\section{Results}

Identification of macro-morphological features, observable with the naked eye (Fig. 1) or with a stereomicroscope (Fig. 2), is the first approach when identifying fungal species. All of the fungi analyzed in this study presented common colony features (Fig. 1): conidial color, mycelial color, and colony diameter. As expected, the common feature, sclerotia, of Aspergillus sclerotioniger MUM 06.151 was 


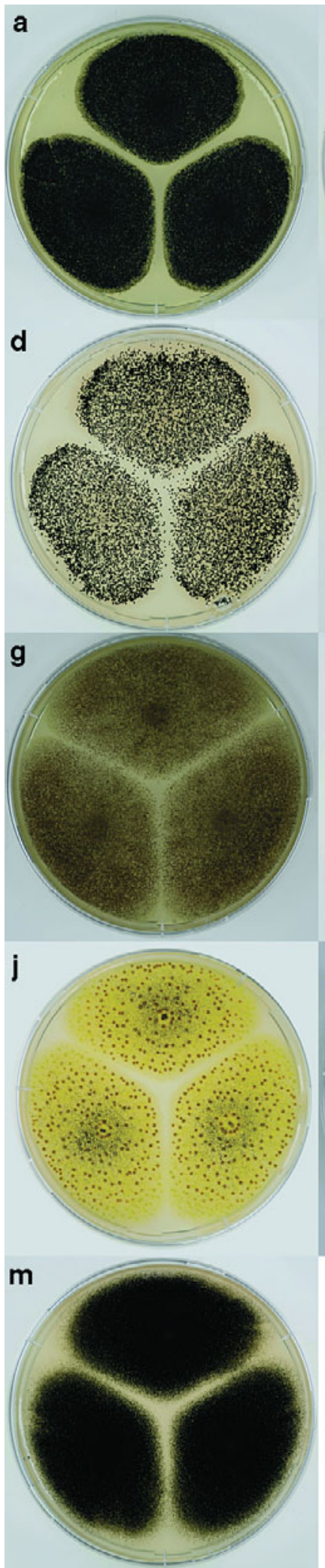

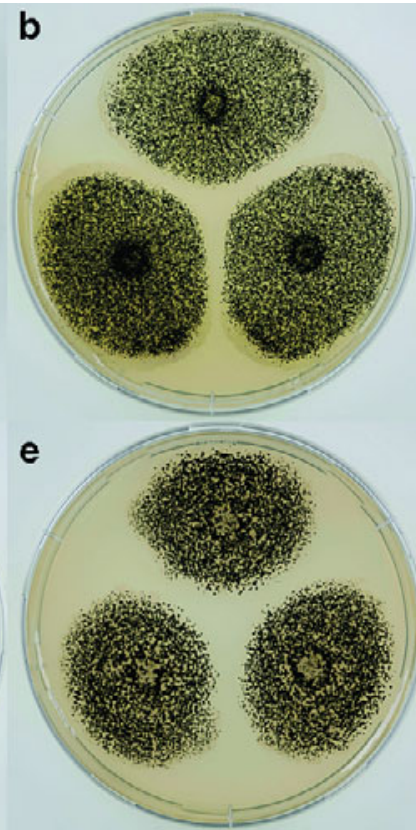

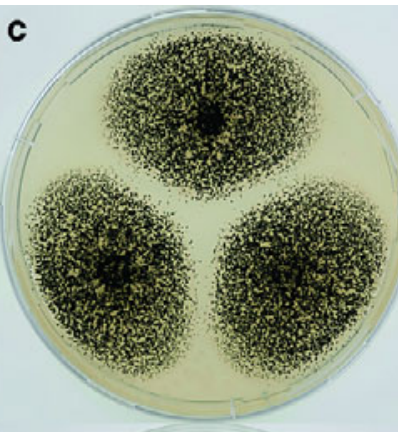

f
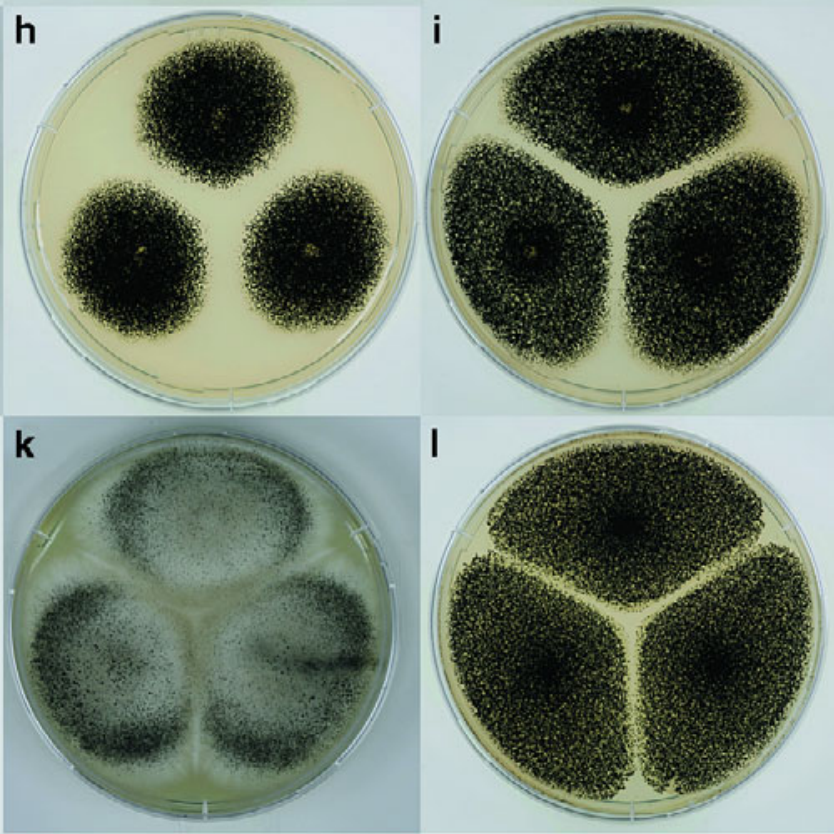

Figure 1. Fungi colonies, grown 7 days in the dark, at $25^{\circ} \mathrm{C}$ in malt extract agar. (a) Aspergillus aculeatus MUM 03.11 ${ }^{\mathrm{T}}$; (b) Aspergillus brasiliensis MUM 06.179; (c) Aspergillus carbonarius MUM 01.08; (d) Aspergillus ellipticus MUM 03.12 ${ }^{\mathrm{T}}$; (e) Aspergillus ibericus MUM 04.68; (f) Aspergillus japonicus MUM 98.03; (g) Aspergillus lacticoffeatus MUM 06.150 ${ }^{\mathrm{T}}$; (h) Aspergillus niger MUM 05.13; (i) Aspergillus phoenicis MUM 03.05; (j) Aspergillus sclerotioniger MUM $06.151^{\mathrm{T}}$; (k) Aspergillus tubingensis MUM $06.152^{\mathrm{T}}$; (l) Aspergillus uvarum MUM 08.01; (m) Aspergillus vadensis MUM $06.153^{\mathrm{T}}$.

also observed (Fig. 1j) (representative data shown for MEA only).

Aspergillus japonicus MUM 98.03 presented poor sporulation (Fig. 2f) when compared with the other strains on both media on which it was grown, but showed its common white-to-cream-colored mycelia with abundant yellow-to- brown soluble pigments on the media more noticeable on the colony reverse (data not shown).

On MEA, conidiophores are largely produced allowing easy retrieval of spores for analysis and characterization. Aspergillus aculeatus, A. japonicus, and Aspergillus uvarum were the only uniseriate species presenting only phialides 


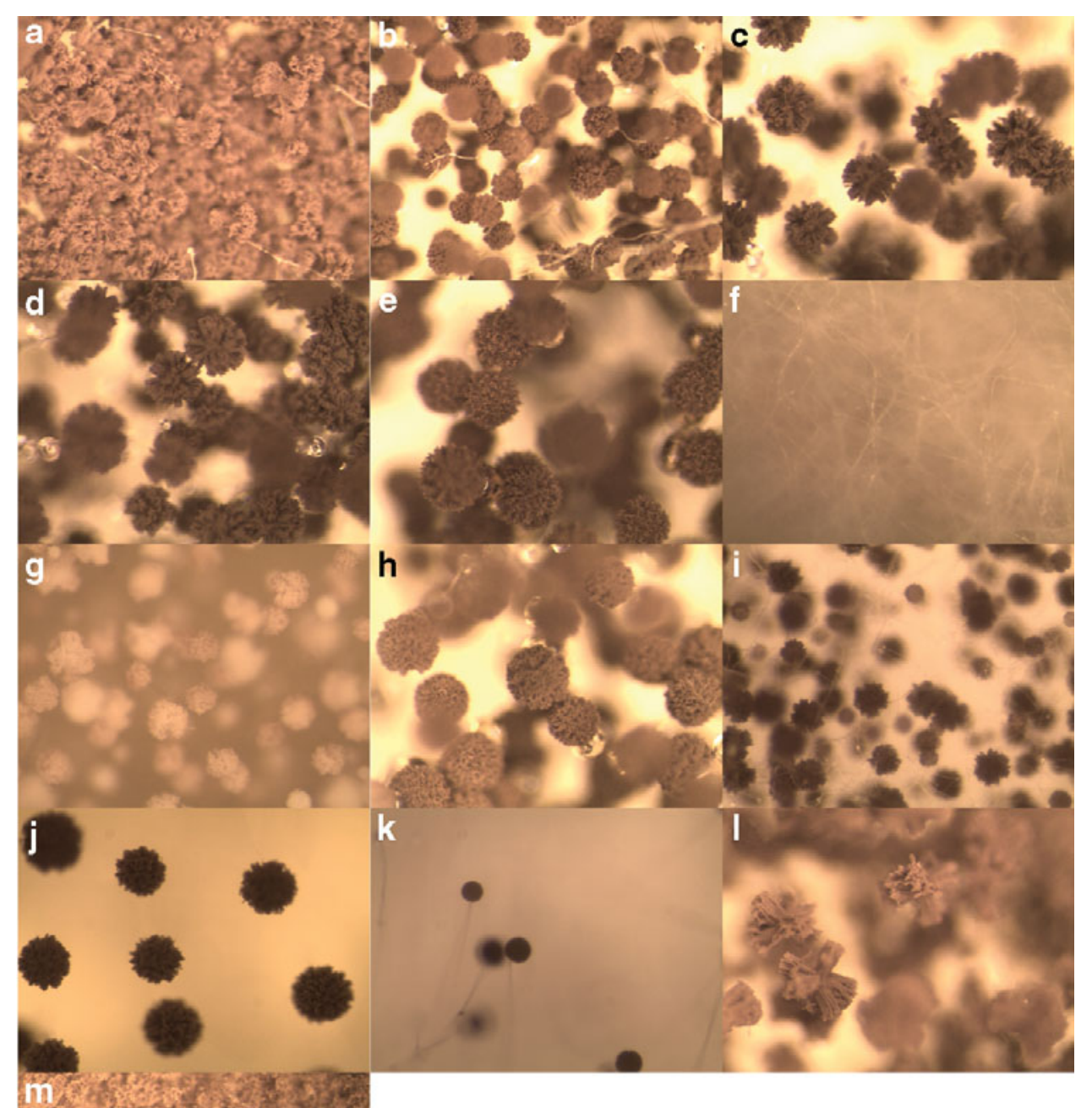

Figure 2. General color and morphology characteristics. (a) Aspergillus aculeatus MUM $03.11^{\mathrm{T}}$; (b) Aspergillus brasiliensis MUM 06.179; (c) Aspergillus carbonarius MUM 01.08; (d) Aspergillus ellipticus MUM 03.12 ${ }^{\mathrm{T}}$; (e) Aspergillus ibericus MUM 04.68; (f) Aspergillus japonicus MUM 98.03; (g) Aspergillus lacticoffeatus MUM 06.150 ${ }^{\mathrm{T}}$; (h) Aspergillus niger MUM 05.13; (i) Aspergillus phoenicis

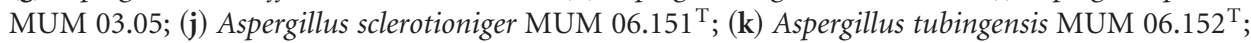
(l) Aspergillus uvarum MUM 08.01; (m) Aspergillus vadensis MUM 06.153 ${ }^{\mathrm{T}}$. Total magnification: $32 \times$.

(Figs. 3a, 3f, 3l); all the other strains presented metulae and phialides (biseriate). Moreover, each strain presented spores with different characteristics (Fig. 4), either in shape or in size of ornamentation features.

From the analysis of the images presented in Figures 3 and 4, the data were composed as shown in Table 2.

Mass spectral analysis allowed for the construction of a dendrogram of relatedness presented in Figure 5.

\section{Discussion}

Phenotypic data are essential for taxonomy. All of the characterization data hereby presented contribute to the increase in associated information of strains belonging to Aspergillus section Nigri. Species belonging to Aspergillus section Nigri usually present dark-brown-to-black spores, with uniseriate or biseriate conidiophores, spherical vesicles, and hyaline or lightly pigmented hyphae
(Klich, 2002). Although the strains evaluated presented dimensional, morphological, and structural diversity (Fig. 2), common or typical features were attributed and related to each taxon, similar to those represented in Figures 3 and 4.

Sclerotia were only detected in A. sclerotioniger (Fig. 1), despite previous reports on the presence of such structures in some of the other strains included in our study (Samson et al., 2004, 2007). This is likely because of variability among cultures of the same isolate as described for some species (Geiser et al., 2007).

It was noticeable that $A$. aculeatus, $A$. japonicus, and $A$. vadensis present the shortest conidiophores (Figs. 2a, 2f, $2 \mathrm{~m})$. Most of the analyzed fungi present globose conidial heads, but $A$. japonicus and $A$. vadensis have radiate conidial heads (Figs. 3f, 3m). Aspergillus ellipticus, Aspergillus ibericus, A. sclerotioniger, and A. uvarum present conidial heads with the largest vesicle (Figs. 3d, 3e, 3j, 3l). Even though 


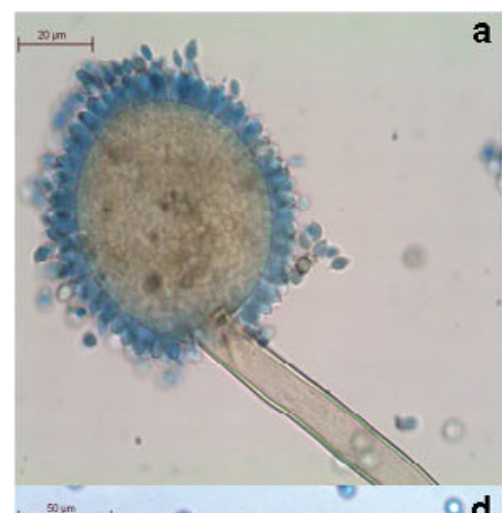

a
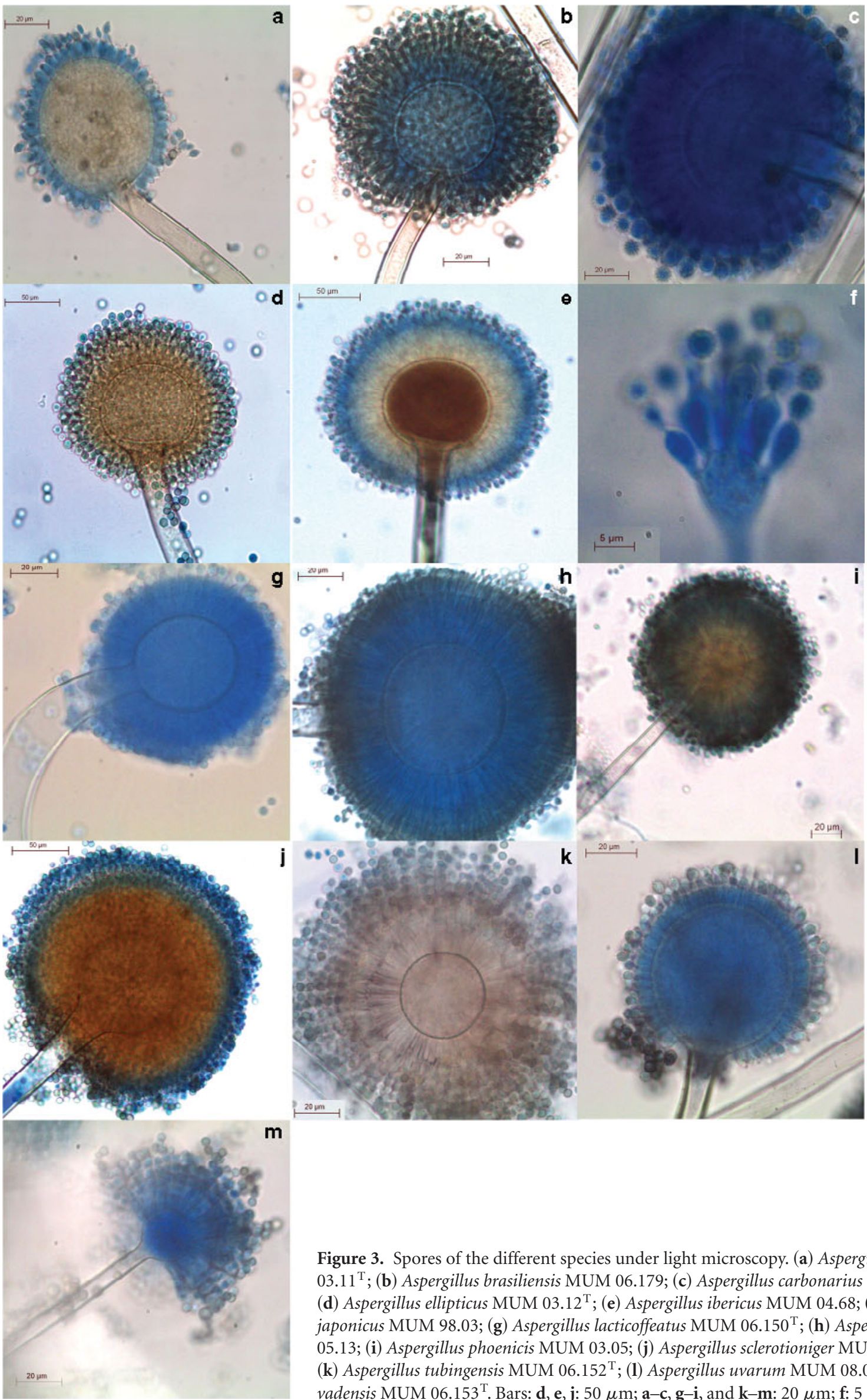

Figure 3. Spores of the different species under light microscopy. (a) Aspergillus aculeatus MUM $03.11^{\mathrm{T}}$; (b) Aspergillus brasiliensis MUM 06.179; (c) Aspergillus carbonarius MUM 01.08; (d) Aspergillus ellipticus MUM 03.12 ${ }^{\mathrm{T}}$; (e) Aspergillus ibericus MUM 04.68; (f) Aspergillus japonicus MUM 98.03; (g) Aspergillus lacticoffeatus MUM 06.150 ${ }^{\mathrm{T}}$; (h) Aspergillus niger MUM 05.13; (i) Aspergillus phoenicis MUM 03.05; (j) Aspergillus sclerotioniger MUM 06.151 ${ }^{\mathrm{T}}$; (k) Aspergillus tubingensis MUM 06.152 ${ }^{\mathrm{T}}$; (1) Aspergillus uvarum MUM 08.01; (m) Aspergillus vadensis MUM 06.153 ${ }^{\mathrm{T}}$. Bars: d, e, j: $50 \mu \mathrm{m}$; $\mathbf{a}-\mathbf{c}, \mathbf{g}-\mathbf{i}$, and $\mathbf{k}-\mathbf{m}: 20 \mu \mathrm{m}$; f: $5 \mu \mathrm{m}$. 

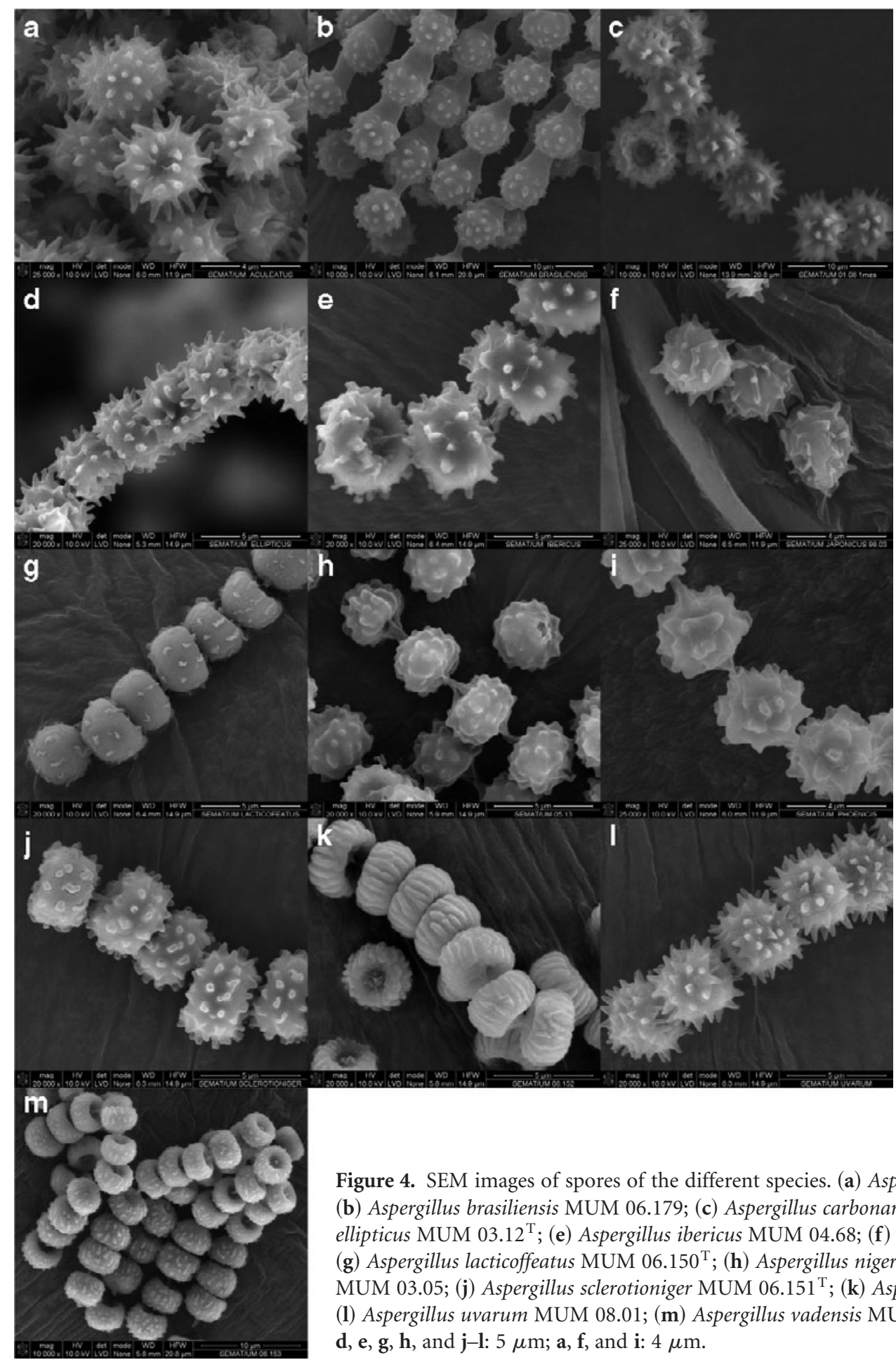

Figure 4. SEM images of spores of the different species. (a) Aspergillus aculeatus MUM $03.11^{\mathrm{T}}$; (b) Aspergillus brasiliensis MUM 06.179; (c) Aspergillus carbonarius MUM 01.08; (d) Aspergillus ellipticus MUM 03.12 $2^{\mathrm{T}}$; (e) Aspergillus ibericus MUM 04.68; (f) Aspergillus japonicus MUM 98.03; (g) Aspergillus lacticoffeatus MUM 06.150 ${ }^{\mathrm{T}}$; (h) Aspergillus niger MUM 05.13; (i) Aspergillus phoenicis MUM 03.05; (j) Aspergillus sclerotioniger MUM 06.151 ${ }^{\mathrm{T}}$; (k) Aspergillus tubingensis MUM 06.152 ${ }^{\mathrm{T}}$; (l) Aspergillus uvarum MUM 08.01; (m) Aspergillus vadensis MUM 06.153 ${ }^{\mathrm{T}}$. Bars: b, c, m: $10 \mu \mathrm{m}$; d, e, g, h, and j-l: $5 \mu \mathrm{m}$; $\mathbf{a}, \mathbf{f}$, and i: $4 \mu \mathrm{m}$.

most vesicles present a globose shape, $A$. aculeatus presents ellipsoidal-shaped vesicles (Fig. 3a).

Within the Aspergillus genus, vesicle ornamentation is used as a primary separation parameter for several sections. However, some species are variable in seriation. It has already been shown by Rodrigues et al. (2007) that this is no longer a mainstay characteristic to differentiate species. Aspergillus parasiticus, for instance, which belongs to a different section but within the same genus, can present itself as uniseriate and in some cases biseriate. Also, most Aspergillus flavus isolates are biseriate but this is not consistent. This is where spore characteristics become a primary taxonomic trait of decision when differentiating morpho-species. When observing Figure 4 it is clear that each strain has a particular spore with different ornamentations, sizes, and shapes.

Perrone et al. (2008), on the basis of morphology, described A. japonicus and A. uvarum as being closely related when compared with the other uniseriates of this 
Table 2. Morphological Characteristics of Each Taxon.

\begin{tabular}{|c|c|c|c|}
\hline Species & $\begin{array}{c}\text { Vesicle } \\
\text { Ornamentation }\end{array}$ & Basic Spore Shape & $\begin{array}{l}\text { Spore Diameter } \\
\quad(\mu \mathrm{m})\end{array}$ \\
\hline A. aculeatus MUM $03.11^{\mathrm{T}}$ & Uniseriate & Equinulate & $4.527( \pm 0.215)$ \\
\hline A. brasiliensis MUM 06.179 & Biseriate & Equinulate & $4.421( \pm 0.182)$ \\
\hline A. carbonarius MUM 01.08 & Biseriate & Equinulate & $7.658( \pm 0.259)$ \\
\hline A. ellipticus MUM $03.12^{\mathrm{T}}$ & Biseriate & Equinulate & $6.997( \pm 0.252)$ \\
\hline A. ibericus MUM 04.68 & Biseriate & Equinulate & $6.205( \pm 0.367)$ \\
\hline A. japonicus MUM 98.03 & Uniseriate & Equinulate & $3.878( \pm 0.245)$ \\
\hline A. lacticoffeatus MUM $06.150^{\mathrm{T}}$ & Biseriate & Punctate & $3.861( \pm 0.315)$ \\
\hline A. niger MUM 05.13 & Biseriate & Equinulate & $3.340( \pm 0.209)$ \\
\hline A. phoenicis MUM 03.05 & Biseriate & Equinulate & $4.044( \pm 0.162)$ \\
\hline A. sclerotioniger MUM $06.151^{\mathrm{T}}$ & Biseriate & Equinulate & $5.553( \pm 0.280)$ \\
\hline A. tubingensis MUM $06.152^{\mathrm{T}}$ & Biseriate & Striate with low ridges & $3.972( \pm 0.252)$ \\
\hline A. uvarum MUM 08.01 & Uniseriate & Equinulate & $4.865( \pm 0.223)$ \\
\hline A. vadensis MUM $06.153^{\mathrm{T}}$ & Biseriate & Verruculose & $4.180( \pm 0.431)$ \\
\hline
\end{tabular}

section. However, for the isolates evaluated in this study there are significant differences between both species: $A$. japonicus has smaller conidial heads (Figs. 3f, 31), with spores with smaller diameter and far less pronounced echines (Figs. 4f, 4l), compared with A. uvarum.

Varga et al. (2007) described Aspergillus brasiliensis as having a unique echinulate spore surface ornamentation. We confirmed this uniqueness (Fig. 4b); however, contrary to the description by these authors, resemblance in shape or size between the spores of $A$. brasiliensis and the spores of $A$. aculeatus and Aspergillus carbonarius was not found. The latter two strains do present a similar shape in spores but they have different diameter sizes (Figs. 4b, 4c), and both are very different from $A$. brasiliensis spores.

The identification of species belonging to the section Nigri has been successfully performed by molecular biology on the basis of multilocus sequence typing, as recently described by Jurjević et al. (2012). Another current technique used for identification of microorganisms is MALDITOF MS (Santos et al., 2010; Rodrigues et al., 2011). The previously published molecular identification strongly agrees with the MALDI-TOF MS results from our study. The individual phenotypic characteristics obtained by SEM analysis of the spores for each species evaluated in the present study were supported by macro-morphological and micromorphological analyses and were concordant with the MALDI-TOF MS results (Fig. 5).

\section{Conclusions}

To develop the macro-morphological, micro-morphological, and mass spectral analyses for phenotypic characterization, 13 strains of Aspergillus section Nigri were selected from a fungal culture collection and evaluated as representative samples for each species. This work presents morphotaxonomy, especially SEM-obtained data, as an attempt to resolve some of the difficulties encountered when identifying the referred fungal section. All of the strains chosen present the same characteristics as described for each type of strain on their first taxonomic description. It is noteworthy that elaborate preparation of SEM samples is unnecessary for the analyzed fungi as already suggested by

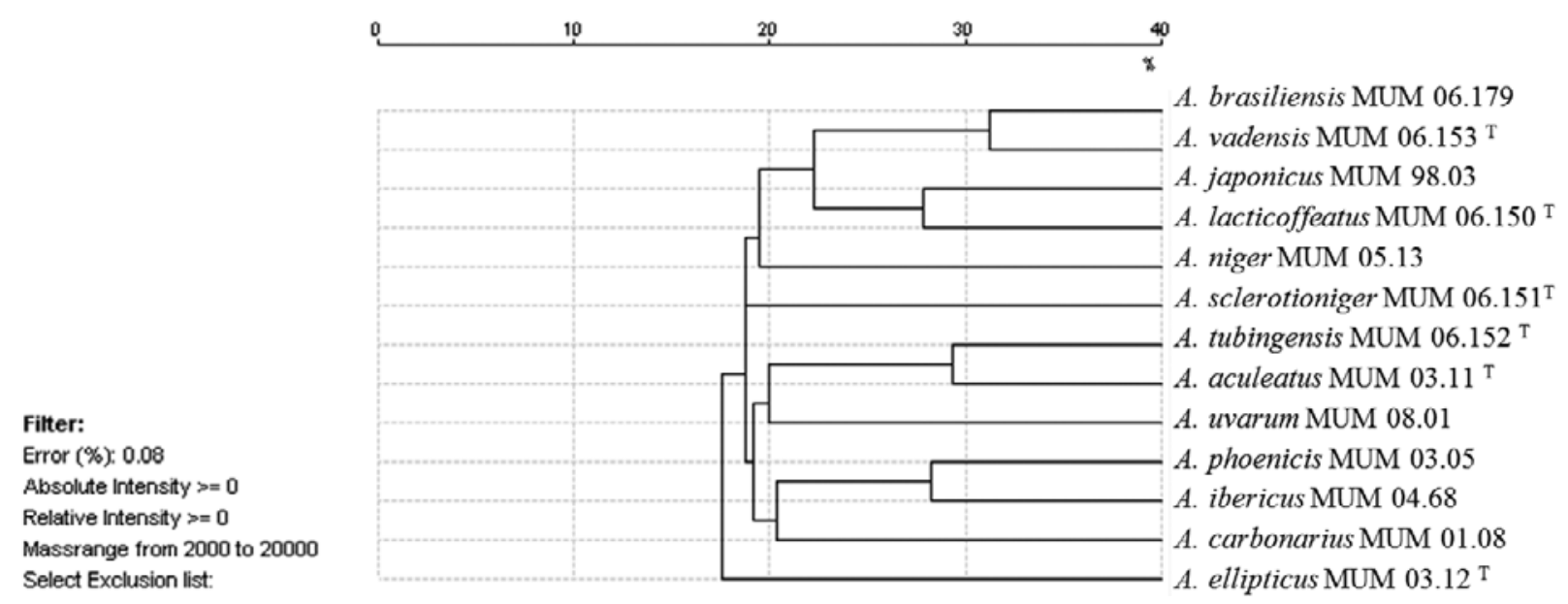

Figure 5. Dendrogram of relatedness between the selected Aspergillus strains of section Nigri based on MALDI-TOF MS analysis. 
Kozakiewicz (1989). From our experience we can say that using complex protocols for this matter deteriorates and collapses the samples.

The size and shape of the fungal spores, as well as their ornamentation, are highly differentiated (Table 2). Although the species investigated presented dimensional, morphological, and structural diversity, common or typical features were inferred and related to each taxon (Figs. 2-4). It is possible to conclude from the phenotypic analysis that within this section the spore wall ornamentation and its size and shape continue to present themselves as important primary diagnostic traits for species differentiation. This can be clearly observed in Figure 4, where spore ornamentation differences are clearly seen. As referred by Serra et al. (2006) and shown in the developed work of Samson et al. (2007), we confirmed that A. carbonarius presents the largest equinulate spores. The smallest spores were observed for Aspergillus niger, Aspergillus lacticoffeatus, and A. japonicus. Aspergillus tubingensis presented the most differentiable conidia with striate spores with low ridges, being the easiest to identify when observing only the spore characteristics. All species differentiation was confirmed and supported by MALDI-TOF MS data (Fig. 5). In conclusion, SEM analysis of fungal spores is a key method to provide information for fungal identification based on morphology.

\section{ACKNOWLEDGMENTS}

This research has received funding from the European Community's Seventh Framework Programme (FP7, 2007-2013), Research Infrastructures action, under grant No. FP7228310 (EMbaRC project). M.F. Simões acknowledges FCTPortugal for the scholarship SFRH/BD/64260/2009.

\section{ReFERENCES}

Abarca, M.L., Accensi, F., Cano, J. \& Cabañes, F.J. (2004). Taxonomy and significance of black aspergilli. Antonie van Leeuwenhoek 86, 33-49.

de Vries, R.P., Frisvad, J.C., van de Vondervoort, P.J., Burgers, K., Kuijpers, A.F., SAmson, R.A. \& Visser, J. (2005). Aspergillus vadensis, a new species of the group of black Aspergilli. Antonie van Leeuwenhoek 87, 195-203.

Geiser, D.M., Klich, M.A., Frisvad, J.C., Peterson, S.W., Varga, J. \& SAmson, R.A. (2007). The current status of species recognition and identification in Aspergillus. Stud Mycol 59, 1-10.

Hubka, V. \& Loralik, M. (2012). $\beta$-tubulin paralogue tubC is frequently misidentified as the benA gene in Aspergillus section Nigri taxonomy: Primer specificity testing and taxonomic consequences. Persoonia 29, 1-10.

Jurjević, Z., Peterson, S.W., Stea, G., Solfrizzo, M., Varga, J., Hubka, V. \& Perrone, G. (2012). Two novel species of Aspergillus section Nigri from indoor air. IMA Fungus 3, 159-173.
KLICH, M.A. (2002). Identification of Common Aspergillus Species. The Netherlands: Centraalbureau voor Schimmelautures.

Kozakiewicz, Z. (1989). Aspergillus Species on Stored Products. Mycological Papers 161. Wallingford, UK: CABI Publishing.

Paterson, R.R.M., Venâncio, A. \& Lima, N. (2004). Solutions to Penicillium taxonomy crucial to mycotoxins research and health. Res Microbiol 155, 507-513.

Paterson, R.R.M., VenÂncio, A. \& Lima, N. (2006). A practical approach for identification based on mycotoxin characters of Penicillium. Rev Iberoam Micol 23, 155-159.

Perrone, G., Varga, J., Susca, A., Frisvad, J., Stea, G., Kocsube, S., Тотн, B., Kozakiewicz, Z. \& Samson, R. (2008). Aspergillus uvarum sp. nov., an uniseriate black Aspergillus species isolated from grapes in Europe. Int J Syst Evol Microbiol 58, 1032-1039.

Rodrigues, P., Santos, C., Venâncio, A. \& Lima, N. (2011). Species identification of Aspergillus section Flavi isolates from Portuguese almonds using phenotypic, including MALDITOF ICMS, and molecular approaches. J Appl Microbiol 111, 877-892.

Rodrigues, P., Soares, C., Kozakiewicz, Z., Paterson, R.R.M., Lima, N. \& VenÂncio, A. (2007). Identification and characterization of Aspergillus flavus and aflatoxins. In Communicating Current Research and Educational Topics and Trends in Applied Microbiology, Méndez-Vilas, A. (Ed.), pp. 527-534. Badajoz, Spain: Formatex.

Samson, R.A., Houbraken, J.A.M.P., Kuijpers, A.F.A., Frank, M.J. \& Frisvad, J.C. (2004). New ochratoxin A or sclerotium producing species in Aspergillus section Nigri. Stud Mycol 50, $45-61$.

Samson, R.A., Noonim, P., Meijer, M., Houbraken, J., Frisvad, J.C. \& Varga, J. (2007). Diagnostic tools to identify black Aspergilli. Stud Mycol 59, 129-145.

Santos, C., Paterson, R.R.M., VenÂncio, A. \& Lima, N. (2010). Filamentous fungal characterizations by matrix-assisted laser desorption/ionization time-of-flight mass spectrometry. J Appl Microbiol 108, 375-385.

Serra, R., Cabañes, F.J., Perrone, G., Castella, G., Venâncio, A., Mule, G. \& Kozakiewickz, Z. (2006). Aspergillus ibericus: A new species of section Nigri isolated from grapes. Mycologia $\mathbf{9 8}$, 295-306.

Simões, M.F., Pereira, L., Santos, C. \& Lima, N. (2013). Polyphasic identification and preservation of fungal diversity: Concepts and applications. In Management of Microbial Resources in the Environment, Malik, A., Grohmann, E. \& Alves, M. (Eds.), pp. 91-118. Dordrecht: Springer.

Varga, J., Frisvad, J.C., Kocsubé, S., Brankovics, B., Tóth, B., Szigeti, G. \& SAmson, R.A. (2011). New and revisited species in Aspergillus section Nigri. Stud Mycol 69, 1-17.

Varga, J., Kocsubé, S., Tóth, B., Frisvad, J.C., Perrone, G., Susca, A., Meijer, M. \& Samson, R.A. (2007). Aspergillus brasiliensis sp. nov., a biseriate black Aspergillus species with world-wide distribution. Int J Syst Evol Microbiol 57, 1925-1932. 